\title{
Pacific
}

Journal of

Mathematics

\section{ON THE COHOMOLOGY OF THE LIE ALGEBRA $L_{2}$}

\section{ALICE FiALOWSKI}

Volume $172 \quad$ No. 2 


\title{
ON THE COHOMOLOGY OF THE LIE ALGEBRA $L_{2}$
}

\author{
Alice Fialowski
}

\begin{abstract}
We compute the 0 -, 1-, and 2-dimensional homology of the vector field Lie algebra $L_{2}$ with coefficients in the modules $\mathcal{F}_{\lambda, \mu}$, and conjecture that the higher dimensional homology for any $\lambda$ and $\mu$ is zero. We completely compute the 0 - and 1-dimensional homology with coefficients in the more complicated modules $F_{\lambda, \mu}$. We also give a conjecture on this homology in any dimension for generic $\lambda$ and $\mu$.
\end{abstract}

\section{Introduction.}

Let us consider the infinite dimensional Lie algebra $W_{1}^{\text {pol }}$ of polynomial vector fields $f(x) d / d x$ on $\mathbb{C}$. It is a dense subalgebra of $W_{1}$, the Lie algebra of formal vector fields on $\mathbb{C}$. We will compute the homology of the polynomial Lie algebra, and will use the notation $W_{1}^{\text {pol }}=W_{1}$. The Lie algebra $W_{1}$ has an additive algebraic basis consisting of the vector fields $e_{k}=x^{k+1} d / d x$, $k \geq-1$, in which the bracket is described by

$$
\left[e_{k}, e_{l}\right]=(l-k) e_{k+l} .
$$

Consider the subalgebras $L_{k}, k \geq 0$ of $W_{1}$, consisting of the fields such that they and their first $k$ derivatives vanish at the origin. The Lie algebra $L_{k}$ is generated by the basis elements $\left\{e_{k}, e_{k+1}, \ldots\right\}$. The algebras $W_{1}$ and $L_{k}$ are naturally graded by $\operatorname{deg} e_{i}=i$. Obviously the infinite dimensional subalgebras $L_{k}$ of $W_{1}$ are nilpotent for $k \geq 1$.

The cohomology rings $H^{*}\left(L_{k}\right), k \geq 0$ with trivial coefficients are known, there exist several different methods for the computation (see $[\mathbf{G}, \mathbf{G F F}$, FF2, FR, V]). The result is the following:

$$
\operatorname{dim} H^{q}\left(L_{k}\right)=\left(\begin{array}{c}
q+k-1 \\
k-1
\end{array}\right)+\left(\begin{array}{c}
q+k-2 \\
k-2
\end{array}\right) \quad \text { for } \quad k \geq 1 .
$$

Not much is known about the cohomology with nontrivial coefficients for the Lie algebra $L_{k}, k>1$. Among the known results, we mention the results on $L_{k}, k \geq 1$ on the cohomology $H^{*}\left(L_{k} ; L_{s}\right)$ with any $s \geq 1$, see [F], and on $L_{k}$, $k \leq 3$ on the cohomology with coefficients in highest weight modules over the Virasoro algebra, see [FF2] and [FF3]. 
Let $F_{\lambda}$ denote the $W_{1}$-module of the tensor fields of the form $f(z) d z^{-\lambda}$, where $f(z)$ is a polynomial in $z$ and $\lambda$ is a complex number; the action of $W_{1}$ on $F_{\lambda}$ is given by the formula

$$
(g d / d x) f d x^{-\lambda}=\left(g f^{\prime}-\lambda f g^{\prime}\right) d x^{-\lambda} .
$$

The module $F_{\lambda}$ has an additive basis $\left\{f_{j} ; j=0,1, \ldots\right\}$ where $f_{j}=x^{j} d x^{-\lambda}$ and the action on the basis elements is

$$
e_{i} f_{j}=(j-(i+1) \lambda) f_{i+j} .
$$

Denote by $\mathcal{F}_{\lambda}$ the $W_{1}$-module which is defined in the same way, except that the index $j$ runs over all integers. The $W_{1}$-modules $F_{\lambda}$ with $\lambda \neq 0$ are irreducible, but as $L_{0}$-modules, they are reducible. For getting an $L_{0^{-}}$ submodule of $F_{\lambda}$, it is enough to take its subspace, generated by $f_{j}, j \geq \mu$, where $\mu$ is a positive integer. Denote the obtained $L_{0}$-module by $F_{\lambda, \mu}$.

More general, let us define the $L_{0}$-module $F_{\lambda, \mu}$ for arbitrary complex number $\mu$, as the space, generated - like $F_{\lambda}$ - by the elements $f_{j}, j=0,1, \ldots$, on which $L_{0}$ acts by

$$
e_{i} f_{j}=(j+\mu-(i+1) \lambda) f_{i+j} .
$$

Finally define the modules $\mathcal{F}_{\lambda, \mu}$ over $W_{1}$ as $F_{\lambda, \mu}$ above, without requiring the positivity of $j$.

The homology of the Lie algebra $L_{1}$ with coefficients in $\mathcal{F}_{\lambda, \mu}$ and $F_{\lambda, \mu}$ are computed in [FF1]. We consider everywhere homology rather than cohomology, but the calculations are more or less equivalent. In the case of $\mathcal{F}_{\lambda, \mu}$ one can use the equality

$$
\left(\mathcal{F}_{\lambda, \mu}\right)^{\prime}=\mathcal{F}_{-1-\lambda,-\mu}
$$

which implies that

$$
H^{q}\left(L_{k} ; \mathcal{F}_{\lambda, \mu}\right)^{\prime}=H_{q}\left(L_{k} ; \mathcal{F}_{-1-\lambda,-\mu}\right) .
$$

In the case of $F_{\lambda, \mu}$ one can use the equality

$$
\left(F_{\lambda, \mu}\right)^{\prime}=\left(\mathcal{F}_{-1-\lambda,-\mu}\right) / F_{-1-\lambda,-\mu}
$$

(see [FF1] for details).

Let us recall the results of [FF1]. Set $e(t)=\left(3 t^{2}+t\right) / 2$ and define the $k$-th parabola $(k=0,1,2, \ldots)$ as a curve on the complex plane with the parametric equation

$$
\lambda=e(t)-1
$$




$$
m-k=e(t)+e(t+k)-1 \text {. }
$$

For $k_{1}, k_{2} \in \mathbb{Z}$ we set

$$
P\left(k_{1}, k_{2}\right)=\left(e\left(k_{1}\right)-1, e\left(k_{1}\right)+e\left(k_{1}\right)-1\right)
$$

and let $\mathbf{P}=\left\{P\left(k_{1}, k_{2}\right): k_{1}, k_{2} \in \mathbb{Z}\right\}$. For a point $P$ of $\mathbf{P}$ let us introduce

$$
k(P)=\left|k_{2}-k_{1}\right|
$$

and

$$
K(P)=\left|k_{1}\right|+\left|k_{2}\right| .
$$

If $P \in \mathbf{P}$, then $K(P) \geq k(P), K(P)=k(P) \bmod 2$ and $P$ lies in the $k(P)$ th parabola. For $k \neq 0$ all the points of the $k$-th parabola with integer coefficients belong to $\mathbf{P}$. On the 0 -th parabola there is one point from $\mathbf{P}$ with $K=0$, and two points with $K=2$, two points with $K=4$, and in general, two points with every even number $K$. For $k \geq 0$ on the $k$-th parabola lie $2 k+2$ points from $\mathbf{P}$ with $K=k$ and four points with $K=k+2$, four with $k+4$, and in general, four with $K=k+2 i$.

Theorem [FF1, Theorem 4.1].

$$
\operatorname{dim} H_{q}^{(m)}\left(L_{1} ; \mathcal{F}_{\lambda, \mu}\right)= \begin{cases}2 & \text { if }(\lambda, \mu+m) \in \mathbf{P} \text { and } K(\lambda, \mu+m)<q \\ 1 & \text { if }(\lambda, \mu+m) \in \mathbf{P} \text { and } K(\lambda, \mu+m)=q \\ 0 & \text { otherwise. }\end{cases}
$$

Corollary. If $\lambda$ is not of the form $e(k)-1$ with $k \in \mathbb{Z}$ and if $\mu \in \mathbb{Z}$, then

$$
H_{*}\left(L_{1} ; \mathcal{F}_{\lambda, \mu}\right)=0
$$

The homology $H_{q}\left(L_{1} ; F_{\lambda, \mu}\right)$ is also computed in [FF1]. We will not formulate the result in details, only some important for us facts.

Theorem (Modification of Theorem 4.2, [FF1]).

1) If $(\lambda, \mu)$ is a generic point so that $(\lambda, \mu+m)$ does not lie on any of the parabolas for any integer $m$, then

$$
H_{*}\left(L_{1} ; F_{\lambda, \mu}\right)=H_{*}\left(L_{2}\right) .
$$

2) If $(\lambda, \mu+j)$ lies on the parabola for some $j$, then $H_{q}\left(L_{1} ; F_{\lambda, \mu}\right)$ is bigger than $H_{1}\left(L_{2}\right)$ at least for some $q$. 
3) In all cases

$$
H_{q}\left(L_{2}\right)=2 q+1 \leq \operatorname{dim} H_{q}\left(L_{1} ; F_{\lambda, \mu}\right) \leq 4 q+1
$$

and the boundaries are reached.

The next problem is to compute homology of $L_{2}$ with coefficients in the modules $\mathcal{F}_{\lambda, \mu}$ and $F_{\lambda, \mu}$. That is the aim of this paper. The results are the following.

Theorem 1.

$$
H_{0}^{(m)}\left(L_{2} ; \mathcal{F}_{\lambda, \mu}\right)= \begin{cases}\mathbb{C} & \text { if } \lambda=-1, m+\mu=-1 \\ 0 & \text { otherwise }\end{cases}
$$

\section{Theorem 2.}

$$
\operatorname{dim} H_{1}^{(m)}\left(L_{2} ; \mathcal{F}_{\lambda, \mu}\right)=\left\{\begin{array}{cc}
2 & \text { if } \lambda=m+\mu=-1 \\
1 & \text { if } \lambda=-1, m+\mu=1,2,3 \\
& \text { or } \lambda=0 \text { and } m+\mu=0 \\
& \text { or } \lambda=1 \text { and } m+\mu=1 \\
0 \quad \text { otherwise. } &
\end{array}\right.
$$

These results are analogous to the ones in [FF1] and one can expect that the picture will be similar for higher homology as well. With this in mind, the following result is a surprise.

\section{Theorem 3.}

$$
\operatorname{dim} H_{2}^{(m)}\left(L_{2} ; \mathcal{F}_{\lambda, \mu}\right)=\left\{\begin{array}{cc}
1 \quad \text { if } \quad \lambda=-1, m+\mu=-1,1,2,3 \\
\\
\text { or } \lambda=0 \text { and } m+\mu=0 \\
\quad \text { or } \lambda=1 \text { and } m+\mu=1 \\
0 \quad \text { otherwise. }
\end{array}\right.
$$

That means that the singular values of the parameters for the two-dimensional homology are the same, as the ones for the one-dimensional homology, which is not the case for the homology of $L_{1}$. Moreover, some partial computational results make the following conjecture plausible.

Conjecture 1. $H_{q}\left(L_{2} ; \mathcal{F}_{\lambda, \mu}\right)=0$ for every $\lambda, \mu$ for $q>2$.

Let us try to explain the behavior of this homology. The main difference of the $L_{2}$ case from the $L_{1}$ case is that $H_{q}\left(L_{1} ; \mathcal{F}_{\lambda, \mu}\right)=0$ for generic $\lambda$ and $\mu$, 
while $H_{q}\left(L_{2} ; \mathcal{F}_{\lambda, \mu}\right)=0$ for all $\lambda$ and $\mu$ (if $q>2$ ). This might have the following explanation. By the Shapiro Lemma (see [CE, Ch. XIII/4, Prop. 4.2]),

$$
H_{q}\left(L_{2} ; \mathcal{F}_{\lambda, \mu}\right)=H_{q}\left(L_{1} ; \operatorname{Ind}_{L_{2}}^{L_{1}} \mathcal{F}_{\lambda, \mu}\right)
$$

and $\operatorname{Ind}_{L_{2}}^{L_{1}} \mathcal{F}_{\lambda, \mu}$ may be regarded as a limit case of the tensor product of modules of the type $F_{\lambda^{\prime}, \mu^{\prime}} \otimes \mathcal{F}_{\lambda, \mu}$. Namely, $\operatorname{Ind}_{L_{2}}^{L_{1}} \mathcal{F}_{\lambda, \mu}=F \otimes \mathcal{F}_{\lambda, \mu}$ where $F$ is the $L_{1}$-module spanned by $g_{j}, j \geq 0$, with the $L_{1}$-action $e_{1} g_{j}=g_{j+1}, e_{i} g_{j}=0$ for $i>1$; the isomorphism is defined by the formula

$$
e_{1}^{k} f_{j} \rightarrow \sum_{m=0}^{k}\left(\begin{array}{c}
k \\
m
\end{array}\right) g_{m} \otimes e_{1}^{k-m} f_{j}
$$

(on the left hand side $e_{1}^{k} f_{j}$ means the action of $e_{1}$ in $\operatorname{Ind}_{L_{2}}^{L_{1}} \mathcal{F}_{\lambda, \mu}$, on the right hand side $e_{1}^{k-m} f_{j}$ means the action of $e_{1}$ in $\left.\mathcal{F}_{\lambda, \mu}\right)$. On the other hand, $F=\lim _{\lambda \rightarrow \infty} F_{\lambda, a \lambda}$ for any $a \neq 2$ : put

$$
g_{j}(\lambda)=(a-2) \lambda((a-2) \lambda+1) \ldots((a-2) \lambda+j-1) f_{j} \in F_{\lambda, a \lambda} ;
$$

then

$$
e_{i} g_{j}(\lambda)=\frac{((a-i-1) \lambda+j) g_{i+j}(\lambda)}{((a-2) \lambda+j) \ldots((a-2) \lambda+j+i-1)}
$$

which tends to the action of $L_{1}$ in $F$ when $\lambda \rightarrow \infty$.

Perhaps the homology

$$
H_{q}\left(L_{1} ; F_{\lambda^{\prime}, \mu^{\prime}} \otimes \mathcal{F}_{\lambda, \mu}\right)
$$

depending not on two but on four parameters, has singular values for some $\lambda, \mu, \lambda^{\prime}, \mu^{\prime}$ for each $q$. The problem of computing the cohomology $H_{q}\left(L_{2} ; \mathcal{F}_{\lambda, \mu}\right)$ is the two-parameter limit version of the previous problem, and it is not surprising that the singular solutions of the first problem have effect on the second problem only for small $q$ values.

Our calculation yields also some results for $H_{*}\left(L_{2} ; F_{\lambda, \mu}\right)$. We will formulate them in Section 3, Theorem 4 and 5.

From Theorem 4 it follows that for generic $\lambda, \mu$,

$$
\operatorname{dim} H_{0}\left(L_{2} ; F_{\lambda, \mu}\right)=2,
$$

and for singular values of $\lambda, \mu, \operatorname{dim} H_{0}\left(L_{2} ; F_{\lambda, \mu}\right)>2$.

From Theorem 5 it follows that for generic $\lambda, \mu$,

$$
\operatorname{dim} H_{1}\left(L_{2} ; F_{\lambda, \mu}\right)=8
$$


and for singular values of $\lambda, \mu, \operatorname{dim} H_{1}\left(L_{2} ; F_{\lambda, \mu}\right)>8$.

Conjecture 2. For generic $\lambda, \mu$,

$$
\operatorname{dim} H_{q}\left(L_{2} ; F_{\lambda, \mu}\right)=2(q+1)^{2}
$$

or in more details,

$$
H_{q}^{(m)}\left(L_{2} ; F_{\lambda, \mu}\right) \simeq H_{q}^{(m)}\left(L_{3}\right) \oplus H_{q}^{(m-1)}\left(L_{3}\right) .
$$

This conjecture is motivated by the following observation. By the Shapiro Lemma,

$$
H_{q}^{(m)}\left(L_{3}\right)=H_{q}^{(m)}\left(L_{2} ; \operatorname{Ind}_{L_{3}}^{L_{2}} \mathbb{C}\right) .
$$

The module $\operatorname{Ind}_{L_{3}}^{L_{2}} \mathbb{C}$ is spanned by $h_{j}(j \geq 0)$ with $L_{2}$-action $e_{2} h_{j}=h_{j+1}$, $e_{i} h_{j}=0$ for $i>2$; the grading in this module is $\operatorname{deg} h_{j}=2 j$. Hence

$$
H_{q}^{(m)}\left(L_{3}\right)=H_{q}^{(m)}\left(L_{2} ; \operatorname{Ind}_{L_{3}}^{L_{2}} \mathbb{C}+\Sigma \operatorname{Ind}_{L_{3}}^{L_{2}} \mathbb{C}\right)
$$

where $\Sigma$ stands for the shift of grading by one. On other words,

$$
H_{q}^{(m)}\left(L_{3}\right) \oplus H_{q}^{(m-1)}\left(L_{3}\right)=H_{q}^{(m)}\left(L_{2} ; F\right)
$$

where $F$ is spanned by $g_{j}, j \geq 0$, with the $L_{2}$-action $e_{2} g_{j}=g_{j+2}, e_{i} g_{j}=0$ for $i>2$. As above, $F=\lim _{\lambda \rightarrow \infty} F_{\lambda, a \lambda}$ (now $a \neq 3$ ), which suggests that

$$
H_{q}^{(m)}\left(L_{2} ; F\right)=H_{q}^{(m)}\left(L_{2} ; F_{\lambda, \mu}\right)
$$

for generic $\lambda, \mu$.

Similarly one can expect that for generic $\lambda, \mu$

$$
H_{q}^{(m)}\left(L_{k} ; F_{\lambda, \mu}\right)=H_{q}^{(m)}\left(L_{k+1}\right) \oplus H_{q}^{(m-1)}\left(L_{k+1}\right) \oplus \cdots \oplus H_{q}^{(m-k+1)}\left(L_{k+1}\right) .
$$

Remark, that if it is true that generically $H_{q}\left(L_{2} ; \mathcal{F}_{\lambda, \mu}\right)=0$ then generically

$$
H^{q}\left(L_{2} ; \mathcal{F}_{\lambda, \mu}\right)=H_{q-1}\left(L_{2} ; F_{-1-\lambda,-\mu}\right)
$$

$\left(H^{q}\left(L_{2} ; \mathcal{F}_{\lambda, \mu}\right)=H_{q}\left(L_{2} ; F_{\lambda, \mu}^{\prime}\right)=H_{q}\left(L_{2} ; \mathcal{F}_{-1-\lambda,-\mu} / F_{-1-\lambda,-\mu}\right)\right.$, and the homology exact sequence associated with the short coefficient exact sequence

$$
0 \rightarrow F_{-1-\lambda,-\mu} \rightarrow \mathcal{F}_{-1-\lambda,-\mu} \rightarrow \mathcal{F}_{-1-\lambda,-\mu} / F_{-1-\lambda,-\mu} \rightarrow 0
$$

provides the above isomorphism). In particular, if the $L_{2}$-module $L_{2}^{\prime}=$ $F_{-2,-3}$ is "generic", then Conjecture 2 implies

$$
\operatorname{dim} H^{2}\left(L_{2} ; L_{2}\right)=\operatorname{dim} H_{1}\left(L_{2} ; F_{-2,-3}\right)=8
$$


Similarly for $L_{k}$ we have the hypothetical result

$$
H^{2}\left(L_{k} ; L_{k}\right)=k(k+2) .
$$

The paper by Yu. Kochetkov and G. Post $[\mathbf{K P}]$ contains the announcement of the equality

$$
\operatorname{dim} H^{2}\left(L_{2} ; L_{2}\right)=8,
$$

as well as some further computations, including explicit formulas for 8 generating cocycles, which imply the description of infinitesimal deformations of the Lie algebra $L_{2}$.

\section{Spectral sequence.}

Let us compute the homology $H_{q}^{(m)}\left(L_{2} ; \mathcal{F}_{\lambda, \mu}\right)$. Define a spectral sequence with respect to the filtration in the cochain complex $C_{*}^{(m)}\left(L_{2} ; \mathcal{F}_{\lambda, \mu}\right)$. The space $C_{q}^{(m)}\left(L_{2} ; \mathcal{F}_{\lambda, \mu}\right)$ is generated by the chains

$$
e_{i_{1}} \wedge \ldots \wedge e_{i_{q}} \otimes f_{j}
$$

where $2 \leq i_{1}<\ldots<i_{q}, j \in \mathbb{Z}$ and $i_{1}+\ldots i_{q}+j=m$. Define the filtration by $i_{1}+\ldots+i_{q}=p$. Denote by $F_{p} C_{q}^{(m)}\left(L_{2} ; \mathcal{F}_{\lambda, \mu}\right)$ the subspace of $C_{q}^{(m)}\left(L_{2} ; \mathcal{F}_{\lambda, \mu}\right)$, generated by monomials of the above form with $i_{1}+\ldots+i_{q} \leq p$. Obviously, $\left\{F_{p} C_{q}^{(m)}\left(L_{2} ; \mathcal{F}_{\lambda, \mu}\right)\right\}_{p}$ is an increasing filtration in the chain complex. The differential acts by the rule

$$
\begin{aligned}
& d\left(e_{i_{1}} \wedge \ldots \wedge e_{i_{q}} \otimes f_{j}\right) \\
& \quad=d\left(e_{i_{1}} \wedge \ldots \wedge e_{i_{q}}\right) \otimes f_{j}-\sum_{s=1}^{q}(-1)^{s} e_{i_{1}} \wedge \ldots \hat{e}_{i_{s}} \wedge \ldots \wedge \ldots e_{i_{q}} \otimes e_{i_{s}} f_{j} .
\end{aligned}
$$

As $m$ is fixed, the filtration in bounded.

Denote the spectral sequence, corresponding to this filtration by $E(\lambda, \mu, m)$. Then we have

$$
E_{0}^{p}=C_{*}^{(p)}\left(L_{2} ; \mathbb{C}\right)
$$

and $d_{0}^{p}$ is the differential $\delta_{p}: C_{*}^{(p)}\left(L_{2} ; \mathbb{C}\right) \rightarrow C_{*-1}\left(L_{2} ; \mathbb{C}\right)$. The first term of the spectral sequence is

$$
E_{1}^{p}=H_{*}^{(p)}\left(L_{2} ; \mathbb{C}\right)
$$

The homology of $L_{2}$ with trivial coefficients is known (see [G]):

$$
H_{q}^{(p)}\left(L_{2}\right)= \begin{cases}\mathbb{C} & \text { if } \frac{3 q^{2}+q}{2} \leq p \leq \frac{3(q+1)^{2}-(q+1)}{2} \\ 0 & \text { otherwise. }\end{cases}
$$


Hence the $E_{1}$ term of our spectral sequence looks as follows:

\begin{tabular}{|l|l|l|l|l|l|l|l|l|l|l|l|l|l|l|l|l|l|}
\hline$H_{0}^{(0)}$ & & $H_{1}^{(2)}$ & $H_{1}^{(3)}$ & $H_{1}^{(4)}$ & & & $H_{2}^{(7)}$ & $H_{2}^{(8)}$ & $H_{2}^{(9)}$ & $H_{2}^{(10)}$ & $H_{2}^{(11)}$ & & & & $H_{3}^{(15)}$ & $H_{3}^{(16)}$ & $\ldots$ \\
\hline
\end{tabular}

where all the spaces $H_{q}^{(p)}$ shown in this diagram are one dimensional.

The spaces $E_{1}^{p}$ do not depend on $\lambda$ and $\mu$, but the differentials of the spectral sequence do. Let us introduce the notation

$$
e_{q}^{ \pm}=\frac{3 q^{2} \pm q}{2}
$$

The differentials

$$
d_{p-r}^{p}: E_{p-r}^{p} \rightarrow E_{p-r}^{r} \quad\left(e_{q}^{+} \leq p<e_{q+1}^{-}, e_{q-1}^{+} \leq r<e_{q}^{-}\right)
$$

form a partial multi-valued mapping $\tilde{\delta}_{q}: H_{q}\left(L_{2}\right) \rightarrow H_{q-1}\left(L_{2}\right)$. We shall define a usual linear operator $\delta_{q}: H_{q}\left(L_{2}\right) \rightarrow H_{q-1}\left(L_{2}\right)$ such that (1) if $\tilde{\delta}_{q}(\alpha)$ is defined for some $\alpha \in H_{q}\left(L_{2}\right)$ then $\delta_{q}(\alpha) \in \tilde{\delta}_{q}(\alpha) ;(2) \delta_{q-1} \circ \delta_{q}=0$. (Certainly, the mapping $\delta_{q}$ will depend on $\lambda, \mu, m$.) Then the limit term of the spectral sequence $E(\lambda, \mu, m)$, that is $H_{*}^{(m)}\left(L_{2} ; \mathcal{F}_{\lambda, \mu}\right)$ will coincide with the homology of the complex

$$
H_{0}\left(L_{2}\right) \stackrel{\delta_{1}}{\leftarrow} H_{1}\left(L_{2}\right) \stackrel{\delta_{2}}{\leftarrow} H_{2}\left(L_{2}\right) \stackrel{\delta_{3}}{\leftarrow} \ldots . .
$$

To define $\delta_{1}, \delta_{2}, \ldots$ we fix for any $q$ and any $p, E_{q}^{+} \leq p<e_{q+1}^{-}$, a cycle $c_{q}^{p} \in C_{q}^{(p)}\left(L_{2}\right)$ which represents the generator of $H_{q}^{(p)}\left(L_{2}\right)$.

It is evident that for each $c_{q}^{p}$ there exist chains

$$
\begin{array}{ll}
b_{q}^{p-u} \in C_{q}^{(p-u)}\left(L_{2}\right), & u \geq 1 \\
g_{q-1}^{v} \in C_{q-1}^{(v)}\left(L_{2}\right), & v<e_{q-1}^{+}
\end{array}
$$

such that

$$
\begin{aligned}
d\left(c_{q}^{p} \otimes f_{m-p}-\sum_{u \geq 1} b_{q}^{p-u} \otimes\right. & \left.f_{m-p+u}\right) \\
& =\sum_{r=e_{q-1}^{+}}^{e_{q}^{-}-1} \alpha_{p, r} c_{q-1}^{r} \otimes f_{m-r}+\sum_{v<e_{q-1}^{+}} g_{q-1}^{v} \otimes f_{m-v}
\end{aligned}
$$

where $\alpha_{p, r}$ are complex numbers depending on $\lambda, \mu, m$. These numbers compose the matrix of some linear mapping $H_{q}\left(L_{2}\right) \rightarrow H_{q-1}\left(L_{2}\right)$, and this mapping is our $\delta_{q}$. 
The chains $b_{q}^{p, u}$ and $g_{q-1}^{v}$ may be chosen in the following way. Since $d c_{q}^{p}=0$, the differential $d\left(c_{q}^{p} \otimes f_{m-p}\right)$ has the form $\sum_{w<p} h_{q-1}^{w} \otimes f_{m-w}$ with $h_{q-1}^{w} \in C_{q-1}^{(w)}\left(L_{2}\right)$. Here the leading term $h_{q-1}^{p-1}$ is a cycle, $d h_{q-1}^{p-1}=0$. Since $H_{q-1}^{p-1}\left(L_{2}\right)=0$, we have $h_{q-1}^{p-1}=d b_{q}^{p-1}$ with $b_{q}^{p-1} \in C_{q}^{(p-1)}\left(L_{2}\right)$. Now, the leading term of $d\left(c_{q}^{p} \otimes f_{m-p}-b_{q}^{p-1} \otimes f_{m-p+1}\right)$. belongs to $C_{q-1}^{(p-1)}\left(L_{2}\right)$ and it is again a cycle. We apply to it the same procedure and do it until the leading term of $d\left(c_{q}^{p} \otimes f_{m-p}-\sum b_{q}^{p-i} \otimes f_{m-p+i}\right)$ belongs to $C_{q-1}^{\left(e_{q}^{-}-1\right)}\left(L_{2}\right)$. This is still a cycle, but it is not necessarily a boundary, for $H_{q-1}^{e_{q}^{-}-1}\left(L_{2}\right) \neq 0$. Now we choose $b_{q}^{e_{q}^{-}-1} \in C_{q}^{\left(e_{q}^{-}-1\right)}\left(L_{2}\right)$ such that $d b_{q}^{e_{q}^{-}-1}$ is our leading term up to some multiple of $c_{q-1}^{e_{q}^{-}-1}$. Then we do the same for $C_{q-1}^{\left(e_{q}^{-}-2\right)}\left(L_{2}\right)$, and so on until we reach $C_{q-1}^{e_{q-1}^{+}-1}\left(L_{2}\right)$.

The matrix $\left|\alpha_{p, r}\right|$ depends on the choice of the cycles $c_{q}^{p}$. It depends also on the particular choice of the chains $b_{q}^{p-u}$, but only up to a triangular transformation. In particular, the kernels and the images of the mappings $\delta_{q}$, and hence the homology $\operatorname{Ker} \delta_{q} / \operatorname{Im} \delta_{q+1}$, are determined by the cycles $c_{q}^{p}$.

Remark that $\operatorname{dim} H_{q}\left(L_{2}\right)=2 q+1$ and hence the matrix of $\delta_{q}$ is a $(2 q-$ $1) \times(2 q+1)$-matrix depending on $\lambda, \mu, m$. We get

$$
\operatorname{dim} H_{q}^{(m)}\left(L_{2} ; \mathcal{F}_{\lambda, \mu}\right)=2 q+1-\operatorname{rank} \delta_{q}-\operatorname{rank} \delta_{q-1} .
$$

II. Computations of $H_{q}^{(m)}\left(L_{2} ; \mathcal{F}_{\lambda, \mu}\right)$.

1. The space $H_{0}^{(m)}\left(L_{2} ; \mathcal{F}_{\lambda, \mu}\right)$.

As the action of $W_{1}$ on $\mathcal{F}_{\lambda, \mu}$ is

$$
e_{i} \otimes f_{j} \rightarrow[j+\mu-\lambda(i+1)] f_{i+j}
$$

and the nontrivial cycles of $H_{1}\left(L_{2}\right)$ are $c_{1}^{2}=e_{2}, c_{1}^{3}=e_{3}, c_{1}^{4}=e_{4}$, the differentials are the following:

$$
\begin{aligned}
& e_{2} \otimes f_{m-2} \rightarrow(m-2+\mu-3 \lambda) f_{m}, \\
& e_{3} \otimes f_{m-3} \rightarrow(m-3+\mu-4 \lambda) f_{m}, \\
& e_{4} \otimes f_{m-4} \rightarrow(m-4+\mu-5 \lambda) f_{m} .
\end{aligned}
$$

The coefficients in the right hand sides depend on $\lambda$ and $m+\mu$, which is natural, because the whole complex $C_{*}^{(m)}\left(L_{2} ; \mathcal{F}_{\lambda, \mu}\right)$ depends only on $\lambda$ and $m+\mu$. On the other hand, there is an isomorphism $\mathcal{F}_{\lambda, \mu}=\mathcal{F}_{\lambda, \mu+1}, f_{\jmath} \rightarrow f_{j+1}$ with the shift of grading by 1 . Therefore we may put $m=0$ and the differential matrix $\delta_{1}: H_{1}\left(L_{2}\right) \rightarrow H_{0}\left(L_{2}\right)$ has the form

$$
(\mu-2-3 \lambda|\mu-3-4 \lambda| \mu-4-5 \lambda) \text {. }
$$


The rank of the matrix is 0 if $\lambda=m=-1$ and 1 in all the other cases. From this it follows

\section{Theorem 1.}

$$
\operatorname{dim} H_{0}^{(m)}\left(L_{2} ; \mathcal{F}_{\lambda, \mu}\right)= \begin{cases}1 & \text { if } \lambda=-1, m+\mu=-1 \\ 0 & \text { otherwise }\end{cases}
$$

2. The space $H_{1}^{(m)}\left(L_{2} ; \mathcal{F}_{\lambda, \mu}\right)$.

The nontrivial cycles of $C_{2}\left(L_{2} ; \mathbb{C}\right)$ are

$$
\begin{aligned}
c_{2}^{7} & =e_{2} \wedge e_{5}-3 e_{3} \wedge e_{4} \\
c_{2}^{8} & =e_{2} \wedge e_{6}-2 e_{3} \wedge e_{5} \\
c_{2}^{9} & =3 e_{2} \wedge e_{7}-5 e_{3} \wedge e_{6} \\
c_{2}^{10} & =e_{2} \wedge e_{8}-3 e_{4} \wedge e_{6} \\
c_{2}^{11} & =5 e_{2} \wedge e_{9}-7 e_{3} \wedge e_{8}
\end{aligned}
$$

of weight $7,8,9,10,11$.

Let us put $\mu-k \lambda-1=A(k, 1)$. Direct calculation shows that

$$
\begin{aligned}
d\left(\left(e_{2} \wedge e_{5}-\right.\right. & \left.\left.3 e_{3} \wedge e_{4}\right) \otimes f_{-7}-A(3,7) e_{2} \wedge e_{3} \otimes f_{-5}\right) \\
= & -3 A(4,7) e_{4} \otimes f_{-4} \\
& +[3 A(5,7)-A(3,7) A(3,5)] e_{3} \otimes f_{-3} \\
& +[-A(6,7)+A(3,7) A(4,5)] e_{2} \otimes f_{-2}
\end{aligned}
$$

hence

$$
\begin{aligned}
\delta_{2}\left(c_{2}^{7}\right)= & {[-A(6,7)+A(3,7) A(4,5)] c_{1} 1^{2} } \\
& +[3 A(5,7)-A(3,7) A(3,5)] c_{1}^{3}-3 A(4,7) c_{1}^{4}
\end{aligned}
$$

Thus we have

$$
\begin{aligned}
& \alpha_{7,2}=-A(6,7)+A(3,7) A(4,5) \\
& \alpha_{7,3}=3 A(5,7)-A(3,7) A(3,5) \\
& \alpha_{7,4}=-3 A(4,7) .
\end{aligned}
$$

In the same way we calculate $\alpha_{p, r}$ for $p=8,9,10,11$ and $r=2,3,4$. We get 
the following $5 \times 3$-matrix:

\begin{tabular}{|c|c|c|}
\hline $\begin{array}{l}A(3,7) A(4,5) \\
\quad-A(6,7)\end{array}$ & $\begin{array}{l}-A(3,7) A(3,5) \\
\quad+3 A(5,7)\end{array}$ & $-3 A(4,7)$ \\
\hline $\begin{array}{c}1 / 2 A(3,8) A(5,6) \\
-2 A(4,8) A(4,5) \\
-A(7,8)\end{array}$ & $\begin{array}{l}2 A(4,8) A(3,5) \\
\quad+2 A(6,8)\end{array}$ & $-1 / 2 A(3,8) A(3,6)$ \\
\hline $\begin{array}{c}-5 / 2 A(4,9) A(5,6) \\
-3 A(8,9)\end{array}$ & $\begin{array}{l}3 A(3,9) A(5,7) \\
\quad+5 A(7,9)\end{array}$ & $\begin{array}{c}-3 A(3,9) A(4,7) \\
+5 / 2 A(4,9) A(3,6)\end{array}$ \\
\hline $\begin{array}{c}-1 / 2 A(3,10) A(4,8) A(4,5) \\
-3 / 2 A(5,10) A(5,6) \\
-A(9,10)\end{array}$ & $\begin{array}{c}1 / 2 A(3,10) A(4,8) A(3,5) \\
\quad+1 / 2 A(3,10) A(6,8)\end{array}$ & $\begin{array}{c}3 / 2 A(5,10) A(3,6) \\
\quad+3 A(7,10)\end{array}$ \\
\hline $\begin{array}{c}7 / 2 A(4,11) A(4,8) A(4,5) \\
+A(3,11) A(8,9) \\
-5 A(10,11)\end{array}$ & $\begin{array}{c}-A(3,11) A(3,9) A(5,7) \\
-7 / 2 A(4,11) A(4,8) A(3,5) \\
-7 / 2 A(4,11) A(6,8) \\
+7 A(9,11)\end{array}$ & $A(3,11) A(3,9) A(4,7)$ \\
\hline
\end{tabular}

We have to compute the rank of the matrix $\left(\delta_{2}\right)$. It is clear that the rank can not be bigger than 2. Direct computation shows that $\operatorname{rk}\left(\delta_{2}\right)=1$ if and only if $\lambda=-1, \mu=-1,1,2,3 ; \lambda=\mu=0 ; \lambda=\mu=1$. From this, using formula $(*)$, it follows

\section{Theorem 2.}

$$
\operatorname{dim} H_{1}^{(m)}\left(L_{2} ; \mathcal{F}_{\lambda, \mu}\right)=\left\{\begin{array}{cc}
2 & \text { if } \lambda=m+\mu=-1 \\
1 & \text { if } \lambda=-1, m+\mu=1,2,3 \\
& \text { or } \lambda=0 \text { and } m+\mu=0 \\
& \text { or } \lambda=1 \text { and } m+\mu=1 \\
0 \quad \text { otherwise. } &
\end{array}\right.
$$

3. The spaces $H_{q}^{(m)}\left(L_{2} ; \mathcal{F}_{\lambda, \mu}\right)$ for $q \geq 2$.

The next differential $\delta_{3}$ is a $5 \times 7$-matrix. Its rank can not be bigger than 3 for any $\lambda$ and $\mu$. On the other hand, computation shows that $\operatorname{rk}\left(\delta_{3}\right)=3$ for every $\lambda, \mu$; namely, the first three rows of the matrix are linearly independent for every $\lambda, \mu$. From this it follows that the dimension of the space $H_{2}^{(m)}\left(L_{2} ; F_{\lambda, \mu}\right)$ drops only if the rank of the previous matrix $\left(\delta_{2}\right)$ does. This proves 


\section{Theorem 3.}

$$
\operatorname{dim} H_{2}^{(m)}\left(L_{2} ; \mathcal{F}_{\lambda, \mu}\right)=\left\{\begin{array}{cc}
1 \quad \text { if } \quad \lambda=-1, m+\mu=-1,1,2,3 \\
\quad \text { or } \lambda=0 \text { and } m+\mu=0 \\
\quad \text { or } \lambda=1 \text { and } m+\mu=1 \\
0 \quad \text { otherwise. }
\end{array}\right.
$$

By this theorem, for generic $\lambda, \mu, \operatorname{dim} H_{2}^{(m)}\left(L_{2} ; \mathcal{F}_{\lambda, \mu}\right)=0$.

It seems very likely that the next differential matrices $\left(\delta_{k}\right), k \geq 4$, have the same rank for every $\lambda$ and $\mu\left(\operatorname{rk}\left(\delta_{k}\right)=q\right)$ which would imply our

Conjecture 1. $H_{q}\left(L_{2} ; \mathcal{F}_{\lambda, \mu}\right)=0$ for every $\lambda, \mu$ for $q>2$.

\section{Computations of $H_{q}^{(m)}\left(L_{2} ; \mathcal{F}_{\lambda, \mu}\right)$.}

Recall that the $L_{0}$-modules $F_{\lambda, \mu}$ differ from the $W_{1}$-modules $\mathcal{F}_{\lambda, \mu}$ only in requiring the non-negativity of $j$ for the generators $f_{j}$. Consequently the spectral sequence is basically the same, only it is truncated as follows:

$$
E_{r}^{p}(\lambda, \mu, m)=0 \quad \text { if } \quad m-p<0 .
$$

The space $C_{q}^{(m)}\left(L_{2} ; F_{\lambda, \mu}\right)$ is generated by the chains

$$
e_{i_{1}} \wedge \ldots \wedge e_{i_{q}} \otimes f_{j}
$$

with $2 \leq i_{1} \leq \ldots \leq i_{q}, j \geq 0$ and $i_{1}+\ldots+i_{q}=m$. This way, for computing homology, we have to compute the rank of truncated matrices, consisting of some of the upper rows of the previous matrices.

Let us compute the space $H_{0}\left(L_{2} ; F_{\lambda, \mu}\right)$. Obviously,

$$
H_{0}^{(0)}\left(L_{2} ; F_{\lambda, \mu}\right)=H_{0}^{(1)}\left(L_{2} ; F_{\lambda, \mu}\right)=\mathbb{C} .
$$

For $m=2$ the differential is the following:

$$
e_{2} \otimes f_{0} \rightarrow(\mu-3 \lambda) f_{2}
$$

which shows that if $\mu=3 \lambda$, then $\operatorname{dim} H_{0}^{(2)}=1$, otherwise $H_{0}^{(2)}\left(L_{2} ; F_{\lambda, \mu}\right)=0$.

For $m>2$

$$
\operatorname{dim} H_{0}^{(m)}\left(L_{2} ; F_{\lambda, \mu}\right)= \begin{cases}1 & \text { if } \lambda=-1 \text { and } m+\mu=-1 \\ 0 & \text { otherwise. }\end{cases}
$$

So we get 


\section{Theorem 4.}

$$
H_{0}^{(m)}\left(L_{2} ; F_{\lambda, \mu}\right)= \begin{cases}\mathbb{C} & \text { if } m=0,1 \\ & \text { or } m=2 \text { and } \mu=3 \lambda \\ & \text { or } \lambda=-1 \text { and } m+\mu=-1 \\ 0 & \text { otherwise. }\end{cases}
$$

Corollary. For generic $\lambda, \mu \quad H_{0}\left(L_{2} ; F_{\lambda, \mu}\right)=2$.

Direct computation proves the result for the space $H_{1}^{(m)}\left(L_{2} ; F_{\lambda, \mu}\right)$.

\section{Theorem 5.}

$\operatorname{dim} H_{1}^{(2)}\left(L_{2} ; F_{\lambda, \mu}\right)= \begin{cases}1 & \text { if } \mu=3 \lambda \\ 0 & \text { otherwise }\end{cases}$ $\operatorname{dim} H_{1}^{(3)}\left(L_{2} ; F_{\lambda, \mu}\right)= \begin{cases}2 & \text { for } \lambda=-1, \mu=-4 \\ 1 & \text { otherwise, }\end{cases}$ $\operatorname{dim} H_{1}^{(4)}\left(L_{2} ; F_{\lambda, \mu}\right)=\operatorname{dim} H_{1}^{(5)}\left(L_{2} ; F_{\lambda, \mu}\right)=\operatorname{dim} H_{1}^{(6)}\left(L_{2} ; F_{\lambda, \mu}\right)$

$$
= \begin{cases}3 & \text { for } \mu=-4, \lambda=-1 \\ 2 & \text { otherwise, }\end{cases}
$$

$\operatorname{dim} H_{1}^{(7)}\left(L_{2} ; F_{\lambda, \mu}\right)= \begin{cases}2 & \text { if } \mu=-8, \lambda=-1 \text { or } \mu=0, \lambda=0 \\ 1 & \text { otherwise, }\end{cases}$

$$
\operatorname{dim} H_{1}^{(8)}\left(L_{2} ; F_{\lambda, \mu}\right)= \begin{cases}2 & \text { if } \mu=-9, \lambda=-1 \\ 1 & \text { for } \lambda \text { and } \mu \text { lying on the curve } \\ & -36 \lambda+147 \lambda^{2}-27 \lambda^{3}+8 \mu-72 \lambda \mu+27 \lambda^{2} \mu \\ & +9 \mu^{2}-9 \lambda \mu^{2}+\mu^{3}=0 \\ 0 & \text { otherwise }\end{cases}
$$

for $m>8, \quad \operatorname{dim} H_{1}^{(m)}\left(L_{2} ; F_{\lambda, \mu}\right)=\operatorname{dim} H_{1}^{(m)}\left(L_{2} ; \mathcal{F}_{\lambda, \mu}\right)$ (see Theorem 2).

Corollary. For generic $\lambda, \mu$, $\operatorname{dim} H_{1}\left(L_{2} ; F_{\lambda, \mu}\right)=8$.

Conjecture 2. For generic $\lambda, \mu$,

$$
\operatorname{dim} H_{q}\left(L_{2} ; F_{\lambda, \mu}\right)=2(q+1)^{2},
$$

or, in more details,

$$
H_{q}^{(m)}\left(L_{2} ; F_{\lambda, \mu}\right) \simeq H_{q}^{(m)}\left(L_{3} ; \mathbb{C}\right) \otimes H_{q}^{(m-1)}\left(L_{3} ; \mathbb{C}\right) .
$$




\section{References}

[CE] H. Cartan and S. Eilenberg, Homological Algebra, Ch. XIII/4, Prop. 4.2., Princeton Univ. Press, 1956.

[F] A. Fialowski, On the comology $H^{*}\left(L_{k} ; L_{s}\right)$, Studia Sci Math. Hung., 27 (1992), 189-200.

[FF1] B.L. Feigin and D.B. Fuchs, Homology of the Lie algebra of vector fields on the line, Funct. Anal. \& Appl., 14 (1980), No. 3, 201-212.

[FF2] - Verma modules over the Virasoro algebra, in Lect. Notes in Math., 1060 (1984), 230-245.

[FF3] Cohomology of some nilpotent subalgebras of the Virasoro and Kac-Moody Lie algebras, J. Geom. and Phys., 5 (1988), No. 2.

[FR] B.L. Feigin and V.S. Retach, On the cohomology of some algebras and superalgebras of vector fields, Uspechi Mat. Nauk, 37 (1982), No. 2, 233-234.

[G] L. Goncharowa, The cohomologies of Lie algebras of formal vector fields on the line, Funct. Anal. \& Appl., 17 (1973), No. 2, 91-97.

[GFF] I.M. Gelfand, B.L. Feigin and D.B. Fuchs, Cohomology of infinite dimensional Lie algebras and the Laplace operator, Funct. Anal. \& Appl., 12 (1978), No. 4, 1-5.

[KP] Yu.Yu. Kochetkov and G.F. Post, Deformations of the infinite dimensional nilpotent Lie algebra $L_{2}$, Funct. Anal. \& Appl., 126 (1992), No. 4, 90-92 (in Russian).

[V] F.V. Veinstein, Filtering bases, cohomology of infinite-dimensional Lie algebras and Laplace operator, Funct. Anal. \& Appl., 119 (1985), No. 4, 11-22.

Received January 20, 1993 and revised May 22, 1993.

UNIVERSITY OF CALIFORNIA

DAVIS, CA 95616-8633

E-mail address: fialowsk@math.ucdavis.edu. 
Rosa M. Miró-Roig, Singular moduli spaces of stable vector bundles on $\mathbf{P}^{3}$

Hitoshi Moriyoshi and Toshikazu Natsume, The Godbillon-Vey Cyclic Cocycle and Longitudinal Dirac Operators

J.C. Naranjo, The positive dimensional fibres of the Prym map

Artur Nicolau and Arne Stray, Nevanlinna's coefficients and Douglas al-

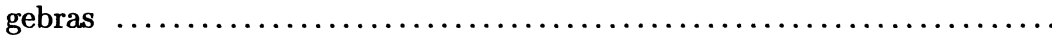

K.K. Park, Entropy of a skew product with a $Z^{2}$-action $\ldots \ldots \ldots \ldots \ldots .227$

María Cristina Pereyra, Sobolev spaces on Lipschitz curves ......... 553

T. Sano, Commuting co-commuting squares and finite dimensional Kac algebras

H.B. Thompson, Second order ordinary differential equations with fully nonlinear two point boundary conditions

H.B. Thompson, Second order ordinary differential equations with fully nonlinear two point boundary conditions II

F. $\mathrm{Xu}$, The flat part of non-flat orbifolds

Hidenobu Yoshida, A type of uniqueness for the Dirichlet problem on a half-space with continuous data 


\section{PACIFIC JOURNAL OF MATHEMATICS}

Volume $172 \quad$ No. $2 \quad$ February 1996

On the failure cycles for the quadratic normality of a projective variety

307

EdoARdo BaLlico

On the minimal free resolution of general embeddings of curves

315

EdOARDO BALlico

On normality of the closure of a generic torus orbit in $G / P$

321

ROMUALD DABROWSKI

Paragroupe d'Adrian Ocneanu et algèbre de Kac

331

MARIE-Claude DAVID

Irreducibility and dimension theorems for families of height 3 Gorenstein algebras

SuSAN J. DiESEL

On the cohomology of the Lie algebra $L_{2}$

ALICE FIALOWSKI

Generic differentiability of convex functions on the dual of a Banach space

John R. Giles, P. S. Kenderov, WarRen Brian Moors and S. D.

SCIFFER

Moon hypersurfaces and some related existence results of capillary hypersurfaces

without gravity and of rotational symmetry

FEI-TSEN LIANG

Stable relations. II. Corona semiprojectivity and dimension-drop $C^{*}$-algebras

TERRY ATHERTON LORING

Singular moduli spaces of stable vector bundles on $\mathbf{P}^{3}$

Rosa M. MIRó-ROIG

The Godbillon-Vey cyclic cocycle and longitudinal Dirac operators

Hitoshi MoRIYOSHI and TOSHIKAZU NATSUME

Nevanlinna's coefficients and Douglas algebras

ARTUR NiCOLAU and ARNE STRAY

Sobolev spaces on Lipschitz curves

MARÍA CRISTINA PEREYRA

A type of uniqueness for the Dirichlet problem on a half-space with continuous data 